\title{
Influência de parâmetros de processo na obtenção de bebida fermento-destilada de uva-japão (Hovenia dulcis Thunberg)
}

Influence of process parameters on obtaining a fermented/distilled drink from the fruit of Hovenia dulcis

\section{Autores | Authors}

Adriano CANCELIER

Universidade Federal de Santa Maria (UFSM) Centro de Tecnologia Departamento de Engenharia Química Santa Maria/RS - Brasil e-mail: adrianocancelier@gmail.com

Catia CAPELETTO Beatriz Alves PEREIRA Diego TODESCATO

Murilo Cesar COSTELLI

Universidade Comunitária da Região de Chapecó (UNOCHAPECO) Área de Ciências Exatas e Ambientais Curso de Engenharia Química Chapecó/SC - Brasil e-mail:katy10@unochapeco.edu.br biaalves@unochapeco.edu.br dtodescato@unochapeco.edu.br mccostell@unochapeco.edu.br

Adriano da SILVA

Fundação Universidade Federal do Rio Grande (FURG)

Santo Antônio da Patrulha/RS - Brasil e-mail: adrianosilva@furg.br

$\square$ Toni Jefferson LOPES

Universidade Federal de Mato Grosso (UFMT)

Instituto de Ciências Exatas e da Terra Rod. MT-100, Km 3,5 CEP: 78698-000

Pontal do Araguaia/MT - Brasil e-mail: tonijl@unochapeco.edu.br

Autor Correspondente / Corresponding Author

Recebido / Received: 02/05/2012 Aprovado / Approved: 03/01/2013 Publicado / Published: mar./2013

\section{Resumo}

Neste trabalho, foi realizado um estudo para avaliar as principais variáveis de processo na produção de uma bebida fermento-destilada a partir do pseudofruto da uva-japão (Hovenia dulcis Thunberg). Foram avaliados os efeitos da temperatura, do tempo de fermentação e da adição de micronutrientes $\left(\mathrm{Mn}^{+2}\right.$ e $\mathrm{K}^{+}$) sobre a graduação alcoólica, além do $\mathrm{pH}$ e dos teores de ésteres presentes nas amostras. A partir dos resultados experimentais obtidos, constatou-se que o pH (pH médio da fração "coração" 5,35) da bebida fermento-destilada a partir da uva-japão não é afetado pelos fatores testados. Para a graduação alcoólica e a concentração de ésteres $\left(2,81 \pm 3,13 \mathrm{mg} \cdot 100 \mathrm{~mL}^{-1}\right)$ presentes na fração "coração" da bebida produzida, os fatores mais significativos foram a temperatura e a concentração de $\mathrm{Mn}^{+2}$. A uva-japão se mostrou uma alternativa viável para a produção de uma bebida fermento-destilada ou como matéria-prima para a produção de bioálcool para usos diversos (95 a $100 \mathrm{GL}$ ).

Palavras-chave: Aguardente; Graduação alcoólica; Planejamento experimental; Nutrientes; Fermentação.

\section{Summary}

A study was carried out to evaluate the main process variables in the production of a fermented/distilled beverage from the pseudofruit of the Japanese grape (Hovenia dulcis Thunberg). The influences of temperature, fermentation time and the addition of micronutrients $\left(\mathrm{Mn}^{+2}\right.$ and $\left.\mathrm{K}^{+}\right)$on the alcoholic graduation, $\mathrm{pH}$ and ester contents of the samples were evaluated. The experimental results showed that the $\mathrm{pH}$ (average $\mathrm{pH}$ value of the "heart fraction" of 5.35) of the fermented/ distilled beverage from the Japanese grape was not affected by the factors tested. The temperature and $\mathrm{Mn}^{+2}$ concentration were the most significant factors influencing the alcoholic graduation and ester content $\left(2.81 \pm 3.13 \mathrm{mg} .100 \mathrm{~mL}^{-1}\right)$ of the "heart fraction" of the beverage produced. The Japanese grape was shown to be a viable alternative for the production of a fermented/distilled beverage or as the raw material to produce bioethanol for diverse applications (95-100 GL).

Key words: Sugarcane spirits; Alcoholic graduation; Experimental design; Nutrients; Fermentation. 


\section{Introdução}

Devido à capacidade que algumas plantas, como a uva-japão (Hovenia dulcis Thunberg), possuem de se adaptar a diferentes climas, há um interesse em explorar o seu cultivo e sua aplicação, pois estas não demandam cuidados significativos. Por apresentar tolerância a geadas e pela multiplicidade de usos, esta espécie tornou-se importante para a Região Sul do Brasil, onde é recomendada para arborização de culturas, pastagens, cerca-viva, uso em serraria e produção de energia por meio da combustão (CARVALHO, 1994).

Considerando-se o elevado teor de açúcares que o pseudofruto da uva-japão possui, cerca de 18,4\% em massa de material seco (SUTTISRI et al., 1995), o mesmo apresenta-se como uma boa alternativa para o desenvolvimento de bebida fermento-destilada, similar à cachaça.

O Brasil está no topo da lista dos países produtores e consumidores de cachaça, apresentando uma produção anual de 1,5 bilhão de litros, equivalente a 11 litros por habitante por ano. Há um aumento crescente na produção de aguardente no país e, pelo fato de sua produção ser, em grande parte, artesanal, surgem algumas limitações no âmbito das exportações (LIMA et al., 2009). A cachaça nada mais é do que uma bebida fermento-destilada que apresenta graduação alcoólica entre 38 e $54 \%$ v. $\mathrm{v}^{-1}$, obtida por meio da fermentação do mosto de cana-deaçúcar e posterior destilação do produto, segundo o Decreto n. ${ }^{0}$ 2314, de 04/09/1997, artigo 91, do Ministério da Agricultura, Pecuária e Abastecimento do Brasil (BRASIL, 1997).

No processo de fermentação alcoólica, os açúcares contidos no caldo de cana são decompostos em álcool etílico e dióxido de carbono, principalmente. Além destes, normalmente há formação de pequenas quantidades de outros componentes, os quais recebem a denominação de produtos secundários da fermentação alcoólica, tais como ácidos carboxílicos, metanol, ésteres, aldeídos e alcoóis superiores (BOGUSZ et al., 2006; SOUZA e DEL MASTRO, 2004; ALCARDE et al., 2010). Destes, os ésteres são compostos de fundamental importância na conferência de odor e sabor às bebidas fermentodestiladas.

Um processo de destilação separa os componentes com base na sua volatilidade. Os compostos mais voláteis, como o metanol e o acetaldeído, são destilados primeiramente, na fração "cabeça". já os menos voláteis, como os alcoóis superiores, são destilados posteriormente nas frações "coração" e "cauda". A parte desejável da cachaça corresponde à fração intermediária (coração), que representa $80-85 \%$ do volume total destilado, é rica em etanol e contém a menor quantidade de compostos secundários que destilam junto com a mistura água-etanol (ROSA et al., 2009).

As frações "cabeça" e "cauda", que constituem $15-20 \%$ do volume total do destilado, são coletadas quando o grau alcoólico atinge $50-70^{\circ} \mathrm{GL}$ e $10-38^{\circ} \mathrm{GL}$, respectivamente (GARCIA-LLOBODANIN et al., 2007; RECHE et al., 2007).

Considerando-se este cenário, o estudo proposto pretende fazer uso da técnica de planejamento experimental para avaliar o efeito de diferentes condições operacionais sobre as características do produto obtido a partir da destilação de mosto fermentado de suco da uva-japão. As condições de processo avaliadas foram a temperatura e o tempo de fermentação, e a adição dos micronutrientes $\mathrm{Mn}^{++} \mathrm{e} \mathrm{K}^{+}$(na forma dos sais $\mathrm{MnSO}_{4} \cdot \mathrm{H}_{2} \mathrm{O}$ e $\mathrm{K}_{2} \mathrm{SO}_{4}$ ). As variáveis avaliadas para descrever as características do produto foram o pH, o teor alcoólico e o teor de ésteres totais.

\section{Material e métodos}

As amostras do pseudofruto da uva-japão foram coletadas em pequenas propriedades rurais, situadas na cidade de Chapecó, no Estado de Santa Catarina, Brasil, nos meses de maio e junho de 2010. Após a coleta, as amostras de uva-japão foram higienizadas para, então, obter-se o suco por processo de prensagem. O suco, depois de filtrado, foi armazenado em freezer a $-5^{\circ} \mathrm{C}$.

Nos ensaios de fermentação, foram utilizados os sais $\mathrm{MnSO}_{4} \cdot \mathrm{H}_{2} \mathrm{O}$ e $\mathrm{K}_{2} \mathrm{SO}_{4}$ (ambos Sigma Aldrich) e o fermento biológico Saccharomyces cerevisiae (Fleischmann), obtidos comercialmente e utilizados sem qualquer tratamento.

\subsection{Fermentação}

Os ensaios de fermentação foram realizados em sistema estático (batelada), em recipientes de $1.000 \mathrm{~mL}$ acondicionados em banho termostático.

O mosto foi obtido a partir de uma diluição do suco da uva-japão em água destilada $\left(50 \% \mathrm{v} \cdot \mathrm{v}^{-1}\right)$, o qual foi pasteurizado a $80^{\circ} \mathrm{C}$ por 20 minutos. Em sequência, o mosto foi resfriado até a temperatura ambiente e filtrado.

O experimento foi iniciado pela adição de $2,0 \mathrm{~g}$ de fermento biológico liofilizado (Saccharomyces cerevisiae) em $1.000 \mathrm{~mL}$ de mosto pasteurizado, o qual já se encontrava na temperatura de fermentação (conforme o planejamento descrito na Tabela 1). Decorrido o tempo de fermentação (Tabela 1), armazenou-se o produto em freezer, à temperatura de $-5{ }^{\circ} \mathrm{C}$, por um período não superior a 24 horas, no qual se realizou o procedimento de destilação.

O efeito de micronutrientes no processo fermentativo foi avaliado pela adição dos íons $\mathrm{Mn}^{++}$e $\mathrm{K}^{+}$(na forma dos sais $\mathrm{MnSO}_{4} \cdot \mathrm{H}_{2} \mathrm{O}$ e $\mathrm{K}_{2} \mathrm{SO}_{4}$ ) ao mosto pasteurizado, 
Influência de parâmetros de processo na obtenção de bebida fermento-destilada de uva-japão (Hovenia dulcis Thunberg) CANCELIER, A. et al.

conforme descrito no planejamento experimental apresentado na Tabela 1.

\subsection{Destilação}

A etapa de destilação fracionada foi realizada em escala de bancada, por meio de um destilador de vidro com capacidade de $1.000 \mathrm{~mL}$. As determinações dos teores de álcool e ésteres totais das amostras de destilado foram efetuadas de acordo com as normas do Instituto Adolfo Lutz (IAL, 2005). Para a determinação do $\mathrm{pH}$, utilizou-se um pHmetro QUIMIS, modelo Q-400A.

\subsection{Influência dos fatores Tempo, Temperatura e} Concentração de Sais sobre a concentração de álcool e ésteres na bebida fermento-destilada de uva-japão

Utilizando-se técnicas de planejamento experimental, avaliou-se a influência de quatro fatores principais (Tabela 1) e suas possíveis interações sobre o processo de obtenção de uma bebida fermento-destilada a partir da uva-japão. Na etapa de triagem dos fatores estudados, optou-se pela utilização de um planejamento fatorial completo $2^{4}$, conforme descrito na Tabela 2.

Posteriormente, na etapa de otimização, optou-se por um planejamento experimental do tipo estrela, com triplicata no ponto central, para os efeitos concentração de $\mathrm{MnSO}_{4} \cdot \mathrm{H}_{2} \mathrm{O}$ e temperatura de fermentação. Na Tabela 1, são mostrados os níveis de variação de cada fator e suas respectivas codificações $(-\alpha,-1,0,+1$ e $+\alpha)$.

\section{Resultados e discussão}

A Tabela 2 mostra a matriz de planejamento fatorial completo $2^{4}$ e as respostas obtidas para cada ensaio, avaliando a influência da temperatura, da concentração de sais e do tempo de fermentação sobre o pH, o teor alcoólico e os ésteres totais da bebida fermento-destilada de uva-japão.

A partir dos resultados apresentados na Tabela 2, foi realizada uma análise estatística utilizando-se

Tabela 1. Fatores e seus respectivos níveis e níveis codificados estudados na produção de álcool e ésteres da bebida fermentodestilada de uva-japão.

\begin{tabular}{|c|c|c|c|c|c|}
\hline \multirow{2}{*}{ Fatores } & \multicolumn{5}{|c|}{ Níveis codificados com os seus respectivos níveis de variação dos fatores } \\
\hline & -1 & 0 & +1 & $-\alpha(-1,41)$ & $+\alpha(+1,41)$ \\
\hline $\mathrm{MnSO}_{4} \cdot \mathrm{H}_{2} \mathrm{O}\left(\mathrm{g} \cdot \mathrm{L}^{-1}\right)$ & 0,031 & 0,077 & 0,123 & 0,012 & 0,142 \\
\hline Temperatura $\left({ }^{\circ} \mathrm{C}\right)$ & 28 & 32 & 36 & 26,3 & 37,6 \\
\hline Tempo de fermentação (horas) & 24 & - & 36 & - & - \\
\hline $\mathrm{K}_{2} \mathrm{SO}_{4}\left(\mathrm{~g} \cdot \mathrm{L}^{-1}\right)$ & 1,56 & - & 1,78 & - & - \\
\hline
\end{tabular}

Tabela 2. Matriz experimental e suas respectivas respostas dos ensaios.

\begin{tabular}{|c|c|c|c|c|c|c|c|c|c|}
\hline \multicolumn{4}{|c|}{ Fatores } & \multicolumn{3}{|c|}{ Resultados referentes à fração "coração" } & \multicolumn{3}{|c|}{ Resultados referentes à fração "cauda" } \\
\hline $\mathbf{A}$ & B & C & D & pH & $\mathbf{E}$ & $\mathbf{F}$ & pH & $\mathbf{E}$ & $\mathbf{F}$ \\
\hline-1 & -1 & -1 & -1 & 5,4 & 74,98 & 3,05 & 3,5 & 10,00 & 60,79 \\
\hline 1 & -1 & -1 & -1 & 5,0 & 67,70 & 3,92 & 3,6 & 15,60 & 27,85 \\
\hline-1 & 1 & -1 & -1 & 5,5 & 78,19 & 3,61 & 3,6 & 13,70 & 19,04 \\
\hline 1 & 1 & -1 & -1 & 5,3 & 72,19 & 4,95 & 3,7 & 6,90 & 3,31 \\
\hline-1 & -1 & 1 & -1 & 5,0 & 74,90 & 15,65 & 3,7 & 28,80 & 3,66 \\
\hline 1 & -1 & 1 & -1 & 5,4 & 79,08 & 3,44 & 3,5 & 4,70 & 9,47 \\
\hline-1 & 1 & 1 & -1 & 5,1 & 72,81 & 3,58 & 3,4 & 9,70 & 11,02 \\
\hline 1 & 1 & 1 & -1 & 5,0 & 72,80 & 2,38 & 3,5 & 6,90 & 18,64 \\
\hline-1 & -1 & -1 & 1 & 5,2 & 70,00 & 1,95 & 3,5 & 3,30 & 72,48 \\
\hline 1 & -1 & -1 & 1 & 4,9 & 68,30 & 3,50 & 3,6 & 13,00 & 13,44 \\
\hline-1 & 1 & -1 & 1 & 5,1 & 77,61 & 1,10 & 3,5 & 4,80 & 31,31 \\
\hline 1 & 1 & -1 & 1 & 5,1 & 71,00 & 3,67 & 3,6 & 12,10 & 10,63 \\
\hline-1 & -1 & 1 & 1 & 4,8 & 75,26 & 3,57 & 3,3 & 8,50 & 7,79 \\
\hline 1 & -1 & 1 & 1 & 5,2 & 77,22 & 2,47 & 3,5 & 7,50 & 46,35 \\
\hline-1 & 1 & 1 & 1 & 5,1 & 72,23 & 2,46 & 3,5 & 12,60 & 0,00 \\
\hline 1 & 1 & 1 & 1 & 5,2 & 72,96 & 4,75 & 3,5 & 10,50 & 24,85 \\
\hline
\end{tabular}

A) $\mathrm{MnSO}_{4} \cdot \mathrm{H}_{2} \mathrm{O}\left(\mathrm{g} . \mathrm{L}^{-1}\right)$; B) $\mathrm{K}_{2} \mathrm{SO}_{4}\left(\mathrm{~g} . \mathrm{L}^{-1}\right)$; C) Temperatura ( $\left.{ }^{\circ} \mathrm{C}\right)$; D) Tempo (h); E) Teor Alcoólico ( $\left.{ }^{\circ} \mathrm{GL}\right)$; F) Esteres totais (mg acetato de etila.100mL ${ }^{-1}$ de álcool anidro). 
Influência de parâmetros de processo na obtenção de bebida fermento-destilada de uva-japão (Hovenia dulcis Thunberg) CANCELIER, A. et al.

ferramentas de planejamento experimental, sendo esta apresentada nos itens a seguir.

\subsection{Determinação da influência dos parâmetros de processo na formação de ésteres totais}

A partir da análise estatística (Figuras 1 e 2), observa-se que a interação da concentração de $\mathrm{MnSO}_{4} \cdot \mathrm{H}_{2} \mathrm{O}$ com a temperatura de fermentação do mosto se mostrou significativa frente aos outros fatores estudados.

Considerando-se que, nas frações "coração" e "cauda", os efeitos mais significativos em relação à quantidade de ésteres foram devidos à interação entre a concentração de $\mathrm{MnSO}_{4} \cdot \mathrm{H}_{2} \mathrm{O}$ e a temperatura, a seguinte etapa realizada foi a de otimização para estes fatores. Neste caso, utilizou-se um planejamento estrela com triplicata no ponto central.

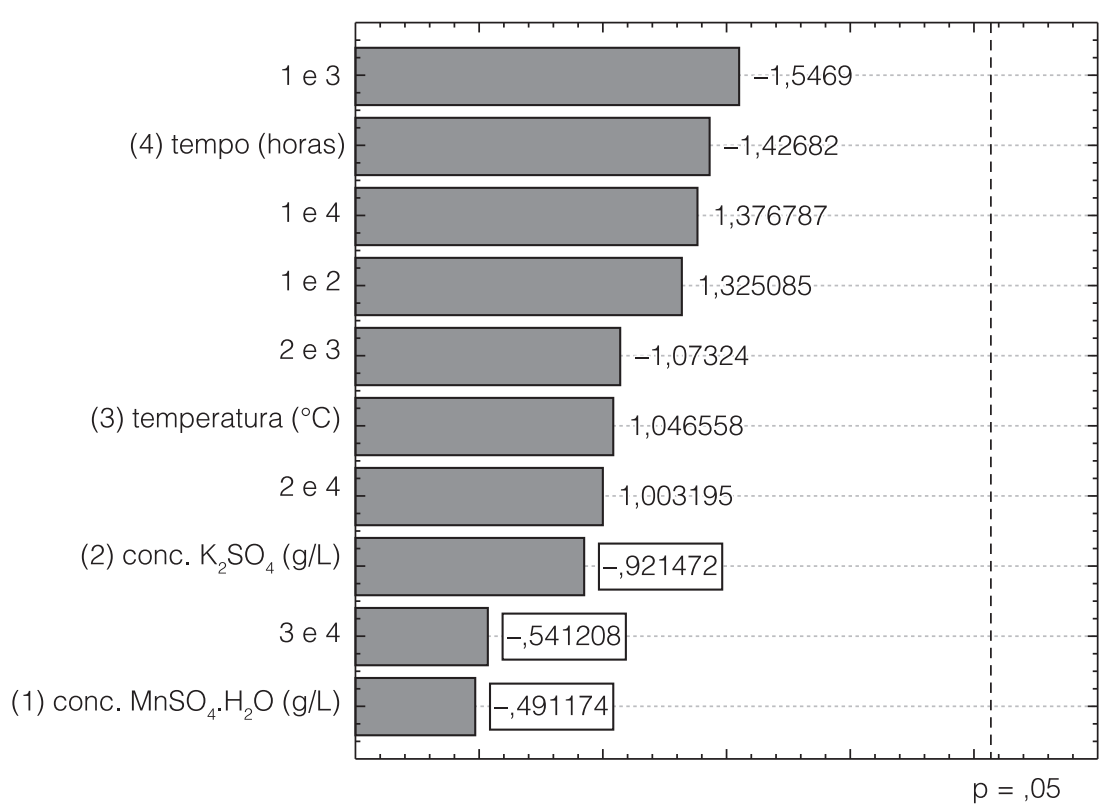

Efeitos estimados padronizados (valor absoluto)

Figura 1. Gráfico de Pareto para a fração "coração" - Esteres totais (mg acetato de etila.100mL-1 de álcool anidro).

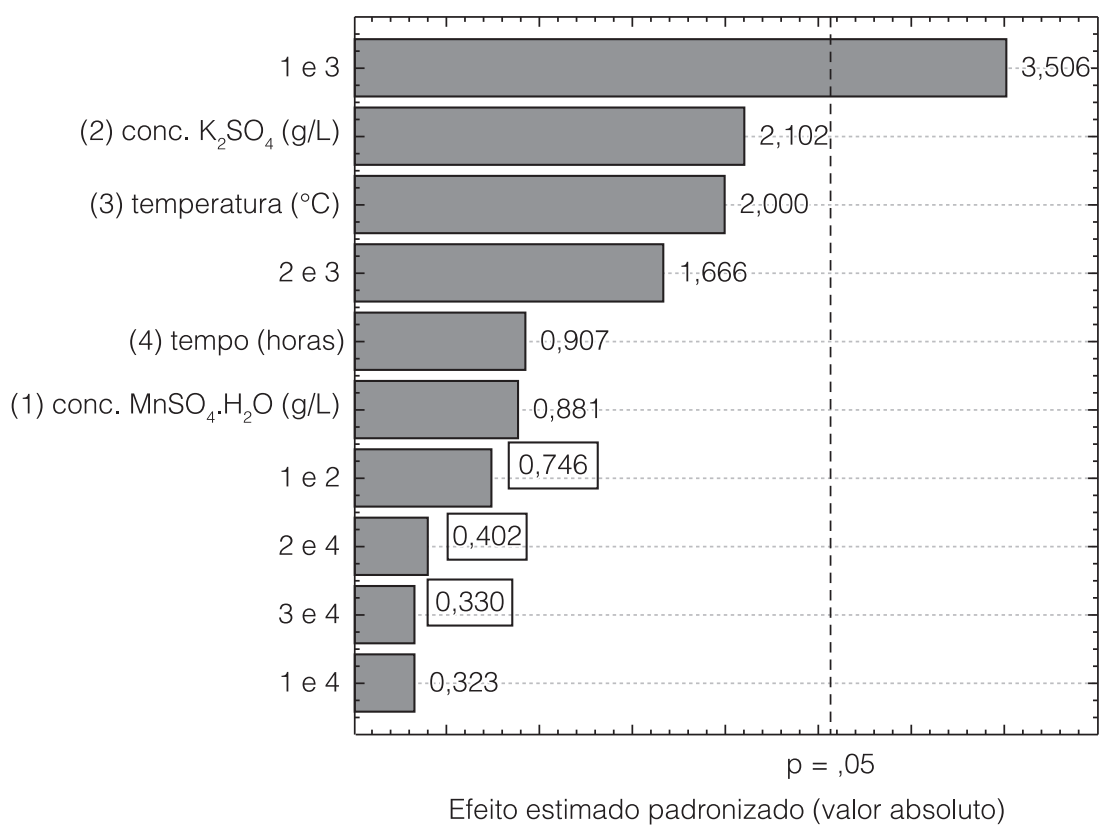

Figura 2. Gráfico de Pareto para a fração "cauda" - Esteres totais (mg acetato de etila. 100mL ${ }^{-1}$ de álcool anidro). 
Influência de parâmetros de processo na obtenção de bebida fermento-destilada de uva-japão (Hovenia dulcis Thunberg) CANCELIER, A. et al.

A Tabela 3 apresenta o planejamento estrela, ou seja, a otimização com suas respectivas respostas.

$\mathrm{Na}$ Tabela 4, apresentam-se os valores obtidos para os efeitos referentes aos fatores concentração de $\mathrm{MnSO}_{4} \cdot \mathrm{H}_{2} \mathrm{O}$ e temperatura referentes à fração "coração", incluindo seus respectivos índices estatísticos.

Analisando-se a Tabela 4, verifica-se que o termo linear ( $\mathrm{L}$ ) da concentração de $\mathrm{MnSO}_{4} \cdot \mathrm{H}_{2} \mathrm{O}$, o termo linear (L) da temperatura e a interação entre os dois termos lineares (1L e 2L) são significativos, como confirmado pelos índices estatísticos apresentados. Também pode-se observar que a concentração de ésteres se comporta inversamente ao comportamento da temperatura, isto é, uma redução da temperatura no processo fermentativo ocasiona um aumento na concentração de ésteres na fração "coração".

Saerens et al. (2008) estudaram a influência de diversas variáveis na fermentação de caldo de canade-açúcar e concluíram que, em uma temperatura de fermentação próxima de $26^{\circ} \mathrm{C}$, há uma maior quantidade de octanoato e decanoato de etila. Segundo Marra (2008), ao utilizar $60 \%$ do caldo de cana-de-açúcar a $30{ }^{\circ} \mathrm{C}$ e o restante, entre 15 e $20^{\circ} \mathrm{C}$, obtém-se maior quantidade de ésteres na fração "coração". Estas observações corroboram com os resultados obtidos neste trabalho, apesar de os estudos terem sido realizados com matériasprimas distintas.
As curvas de nível correspondentes à superfície de resposta gerada pelo modelo linear, que descreve o comportamento da concentração de ésteres em relação aos fatores temperatura e concentração de sulfato de manganês podem ser observadas na Figura 3. Nesta, verifica-se que a concentração de ésteres aumenta com a redução da temperatura e com o aumento da concentração de $\mathrm{MnSO}_{4} \cdot \mathrm{H}_{2} \mathrm{O}$. O nível máximo da concentração de ésteres corresponde à região delimitada pelo fator temperatura variando de 30 a $24^{\circ} \mathrm{C}$ (níveis $-0,5$ a $-2,0$ ) e pelo fator concentração de $\mathrm{MnSO}_{4} \cdot \mathrm{H}_{2} \mathrm{O}$ variando de 0,114 a 0,169 g.L-1 (níveis 0,8 a 2,0).

O controle do processo de destilação deve levar em conta critérios como a eliminação de compostos tóxicos, mas ao mesmo tempo evitar a eliminação de constituintes que conferem sabor e assegurar que o teor de etanol fique dentro dos limites estabelecidos (CARDEAL et al., 2008). Por isso, a quantidade de ésteres não pode ser muito baixa, a ponto de comprometer as características organolépticas, nem tão alta, de modo a ultrapassar o limite de 200 mg.100 $\mathrm{mL}^{-1}$ álcool anidro.

Normalmente, as frações de "cabeça" e "cauda" são descartadas por apresentarem altos teores de aldeídos (furfural, hidroximetilfurfural etc), álcool, cobre e alcoóis superiores (óleo fúsel), que são considerados tóxicos e reduzem substancialmente o rendimento industrial e o valor sensorial. O carbamato de etila é um

Tabela 3. Matriz do planejamento estrela com triplicata no ponto central e suas respectivas respostas.

\begin{tabular}{cccc} 
Concentração de $\mathrm{MnSO}_{\mathbf{4}} \mathbf{H}_{\mathbf{2}} \mathbf{O}$ & Temperatura & $\begin{array}{c}\text { Ésteres totais (mg acetato de etila.100 } \mathbf{m L}^{-1} \text { álcool anidro) } \\
\text { Fração "cauda" }\end{array}$ \\
-1 & -1 & 1,88 & 0,96 \\
1 & -1 & 11,89 & 2,56 \\
-1 & 1 & 1,38 & 1,86 \\
1 & 1 & 1,94 & 1,08 \\
$-1,41$ & 0 & 0,00 & 0,60 \\
1,41 & 0 & 2,25 & 1,70 \\
0 & $-1,41$ & 1,50 & 5,00 \\
0 & 1,41 & 1,31 & 2,31 \\
0 & 0 & 2,86 & 0,16 \\
0 & 0 & 2,86 & 2,49 \\
\hline
\end{tabular}

Tabela 4. Análise estatística dos efeitos sobre os ésteres totais (mg acetato de etila.100 $\mathrm{mL}^{-1}$ de álcool anidro), correspondente à fração "coração" (R² $=61,41 \%)$.

\begin{tabular}{lccccc}
\multicolumn{1}{c}{ Fator } & Efeito & Desvio padrão & $\mathbf{P}$ & $\begin{array}{c}\mathbf{- 9 5 \%} \\
\text { Limite de confiança }\end{array}$ & $\begin{array}{c}\mathbf{+ 9 5 \%} \\
\text { Limite de confiança }\end{array}$ \\
\hline Média/Interações & 2,81 & 0,033 & 0,00013 & 2,67 & 2,95 \\
$(1) \mathrm{MnSO}_{4} \cdot \mathrm{H}_{2} \mathrm{O}\left(\mathrm{g} \cdot \mathrm{L}^{-1}\right)(\mathrm{L})$ & 3,44 & 0,078 & 0,00050 & 3,11 & 3,78 \\
$(2) \mathrm{Temperatura}\left({ }^{\circ} \mathrm{C}\right)(\mathrm{L})$ & $-2,69$ & 0,078 & 0,00083 & $-3,02$ & $-2,35$ \\
$(1)(\mathrm{L})$ e $(2)(\mathrm{L})$ & $-4,72$ & 0,110 & 0,00053 & $-5,20$ & $-4,25$ \\
\hline
\end{tabular}




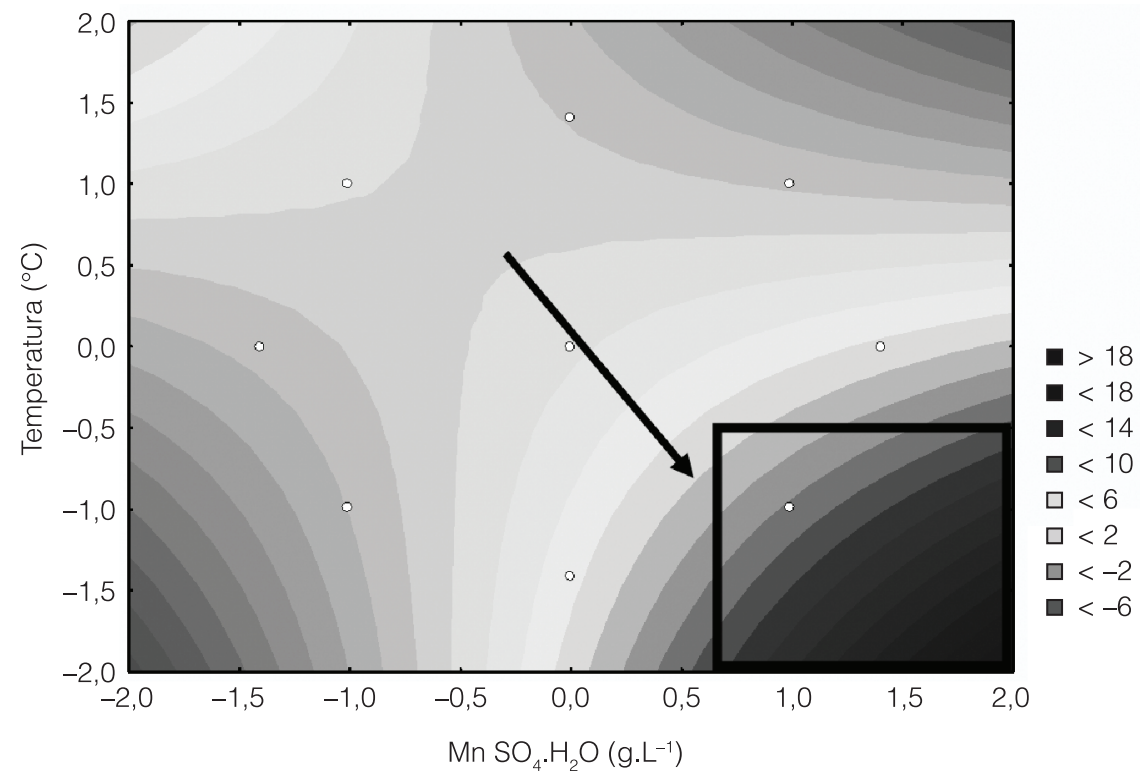

Figura 3. Variação da concentração de ésteres em relação aos fatores temperatura e concentração de $\mathrm{MnSO}_{4} \mathrm{H}_{2} \mathrm{O}$.

composto que tem mostrado um potencial altamente cancerígeno e a sua formação é influenciada por fatores, como $\mathrm{pH}$, luz, quantidade de etanol, temperatura e teor de cobre (ARESTA et al., 2001).

Assim, considerando-se a produção de bebida fermento-destilada, a fração de maior importância é, sem dúvida, a fração "coração". Em razão disso, foi realizada a análise nesta fração, tendo sido obtido um valor médio de 2,81 mg acetato de etila.100 $\mathrm{mL}^{-1}$ de álcool anidro, correspondentes à fração "coração". Este valor encontra-se muito abaixo do permitido pela legislação;

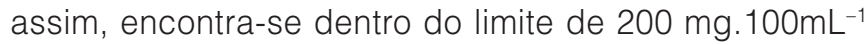
álcool anidro estabelecido pelo MAPA do Brasil (Ministério da Agricultura, Pecuária e Abastecimento) (BRASIL, 1997).

O valor médio de ésteres ficou abaixo dos valores encontrados por Alcarde et al. (2010), que obteve na primeira amostra um valor de $71,95 \mathrm{mg}$ acetato de etila. $100 \mathrm{~mL}^{-1}$ de álcool anidro, e também do valor médio obtido por Dantas et al. (2007), de 24,43 mg.100 $\mathrm{mL}^{-1}$ de álcool anidro, ambos os estudos utilizando cana-deaçúcar como matéria-prima. O valor obtido neste estudo ficou mais próximo do valor médio apresentado por Miranda et al. (2008) de 7,88 mg.100 $\mathrm{mL}^{-1}$ álcool anidro, obtido para a cana-de-açúcar como fonte de açúcar.

\subsection{Determinação da influência dos parâmetros de processo no teor alcoólico e no pH}

A análise estatística, realizada com os dados apresentados na Tabela 2, demonstrou que, para as frações "cauda" e "coração", não houve efeitos significativos para os fatores e suas interações que resultassem em variações de maior expressão no $\mathrm{pH}$.
Ainda, para a fração "cauda", não foi verificado nenhum fator significativo para variações do teor alcoólico. Assim, considerando-se estes resultados e o fato de que a fração de maior importância, para a produção de bebida fermento-destilada, é a fração "coração", realizou-se a análise desta fração para o teor alcoólico.

$\mathrm{Na}$ Tabela 5, apresentam-se os valores obtidos para os efeitos referentes aos fatores concentração de $\mathrm{MnSO}_{4} \cdot \mathrm{H}_{2} \mathrm{O}$, concentração de $\mathrm{K}_{2} \mathrm{SO}_{4}$, tempo e temperatura, e seus respectivos índices estatísticos.

Observa-se, na Tabela 5, que os efeitos principais foram promovidos pela concentração de $\mathrm{MnSO}_{4} \mathrm{H}_{2} \mathrm{O}$ (1) e pela temperatura (3), além das interações entre (1) e (2) e (2) e (3), as quais se mostraram mais significativas frente aos outros fatores estudados. Ou seja, dentro do limite de confiança adotado, não apresenta, no seu intervalo, o valor zero, ou efeito nulo na resposta, o que não ocorre com os outros fatores e suas interações. Também, pode-se observar que a temperatura apresenta um valor de efeito positivo, indicando que a variável resposta é diretamente proporcional à temperatura, ou seja, um acréscimo da temperatura do sistema provoca um aumento no teor alcoólico obtido. Portanto, pode-se dizer que a temperatura de fermentação afeta diretamente o metabolismo dos microrganismos do mosto (leveduras).

Torija et al. (2003) encontraram os seguintes efeitos: com o aumento da temperatura, o teor de álcool diminuiu, porém, a quantidade de metabólitos secundários aumentou. A Tabela 6 apresenta o planejamento estrela para a variação do teor alcoólico na fração "coração" na etapa de otimização.

$\mathrm{Na}$ Tabela 7, apresentam-se os valores obtidos para os efeitos referentes aos fatores concentração de 
Influência de parâmetros de processo na obtenção de bebida fermento-destilada de uva-japão (Hovenia dulcis Thunberg) CANCELIER, A. et al.

Tabela 5. Efeitos significativos dos fatores avaliados sobre o teor de álcool da fração "coração".

\begin{tabular}{|c|c|c|c|c|c|}
\hline Fator & Efeito & Desvio padrão & $\mathbf{P}$ & $\begin{array}{c}-0,95 \% \\
\text { Limite confiança }\end{array}$ & $\begin{array}{c}0,95 \% \\
\text { Limite confiança }\end{array}$ \\
\hline Média/Interações & 73,58 & 0,33 & 0,0000 & 72,72 & 74,43 \\
\hline (1) $\mathrm{MnSO}_{4} \mathrm{H}_{2} \mathrm{O}\left(\mathrm{g} \cdot \mathrm{L}^{-1}\right)$ & $-1,84$ & 0,66 & 0,0393 & $-3,55$ & $-0,13$ \\
\hline$(2) \mathrm{K}_{2} \mathrm{SO}_{4}\left(g \cdot \mathrm{L}^{-1}\right)$ & 0,29 & 0,66 & 0,6769 & $-1,41$ & 2,00 \\
\hline (3) Temperatura $\left({ }^{\circ} \mathrm{C}\right)$ & 2,16 & 0,66 & 0,0226 & 0,45 & 3,87 \\
\hline (4)Tempo (horas) & $-1,01$ & 0,66 & 0,1895 & $-2,72$ & 0,70 \\
\hline (1) e (2) & $-1,13$ & 0,66 & 0,1494 & $-2,84$ & 0,58 \\
\hline (1) e (2) & 3,56 & 0,66 & 0,0031 & 1,85 & 5,26 \\
\hline (1) e (4) & 0,44 & 0,66 & 0,5405 & $-1,27$ & 2,14 \\
\hline (2) e (3) & $-4,21$ & 0,66 & 0,0014 & $-5,92$ & $-2,50$ \\
\hline (2) e (4) & 0,46 & 0,66 & 0,5185 & $-1,25$ & 2,17 \\
\hline (3) e (4) & 0,53 & 0,66 & 0,4623 & $-1,18$ & 2,24 \\
\hline
\end{tabular}

Tabela 6. Matriz planejamento estrela com triplicata no ponto central com suas respectivas respostas.

\begin{tabular}{|c|c|c|}
\hline Concentração de $\mathrm{MnSO}_{4} \cdot \mathrm{H}_{2} \mathrm{O}$ & Temperatura & *Teor alcoólico (GL) \\
\hline-1 & -1 & 71,84 \\
\hline 1 & -1 & 56,30 \\
\hline-1 & 1 & 65,60 \\
\hline 1 & 1 & 71,56 \\
\hline$-1,41$ & 0 & 69,30 \\
\hline 1,41 & 0 & 76,60 \\
\hline 0 & $-1,41$ & 64,20 \\
\hline 0 & 1,41 & 74,68 \\
\hline 0 & 0 & 56,60 \\
\hline 0 & 0 & 59,80 \\
\hline 0 & 0 & 47,50 \\
\hline
\end{tabular}

*Resultados referentes à fração "coração".

Tabela 7. Análise estatística dos efeitos para os teores alcoólicos (GL), correspondente à fração "coração" $\left(R^{2}=77,44 \%\right)$.

\begin{tabular}{|c|c|c|c|c|c|}
\hline & Efeito & Desvio padrão & p & $\begin{array}{l}-95 \% \\
\text { Limite de confiança }\end{array}$ & $\begin{array}{c}+95 \% \\
\text { Limite de confiança }\end{array}$ \\
\hline Média/Interações & 54,65 & 3,50 & 0,000020 & 45,64 & 63,66 \\
\hline (1) $\mathrm{MnSO}_{4} \cdot \mathrm{H}_{2} \mathrm{O}\left(\mathrm{g} \cdot \mathrm{L}^{-1}\right)(\mathrm{L})$ & 0,18 & 4,30 & 0,968442 & $-10,87$ & 11,23 \\
\hline $\mathrm{MnSO}_{4} \cdot \mathrm{H}_{2} \mathrm{O}\left(\mathrm{g} \cdot \mathrm{L}^{-1}\right)(\mathrm{Q})$ & 15,91 & 5,13 & 0,026839 & 2,72 & 29,09 \\
\hline (2) Temperatura $\left({ }^{\circ} \mathrm{C}\right)(\mathrm{L})$ & 5,97 & 4,30 & 0,223869 & $-5,08$ & 17,02 \\
\hline Temperatura $\left({ }^{\circ} \mathrm{C}\right)(\mathrm{Q})$ & 12,38 & 5,13 & 0,060697 & $-0,81$ & 25,56 \\
\hline$(1)(L)$ e (2) $(L)$ & 10,75 & 6,07 & 0,136850 & $-4,86$ & 26,36 \\
\hline
\end{tabular}

$\mathrm{MnSO}_{4} \cdot \mathrm{H}_{2} \mathrm{O}$ e temperatura, e seus respectivos índices estatísticos.

Analisando-se a Tabela 7, verifica-se que o termo quadrático $(\mathrm{Q})$ da concentração de $\mathrm{MnSO}_{4} \cdot \mathrm{H}_{2} \mathrm{O}$ é significativo, como confirmado pelos índices estatísticos apresentados. Também pode-se observar que um aumento da temperatura ou da concentração do sal no processo fermentativo ocasiona um aumento no teor alcoólico na fração "coração".
As curvas de nível, correspondentes à superfície de resposta gerada pelo modelo linear/quadrático com interações, que descreve o comportamento do teor alcóolico em relação aos fatores temperatura e concentração de $\mathrm{MnSO}_{4} \cdot \mathrm{H}_{2} \mathrm{O}$ para a fração "coração", podem ser observadas na Figura 4.

Verifica-se, na Figura 4, que nos extremos da concentração de sal de manganês há um maior teor alcoólico do destilado de uva-japão. A região de menor 
Influência de parâmetros de processo na obtenção de bebida fermento-destilada de uva-japão (Hovenia dulcis Thunberg) CANCELIER, A. et al.

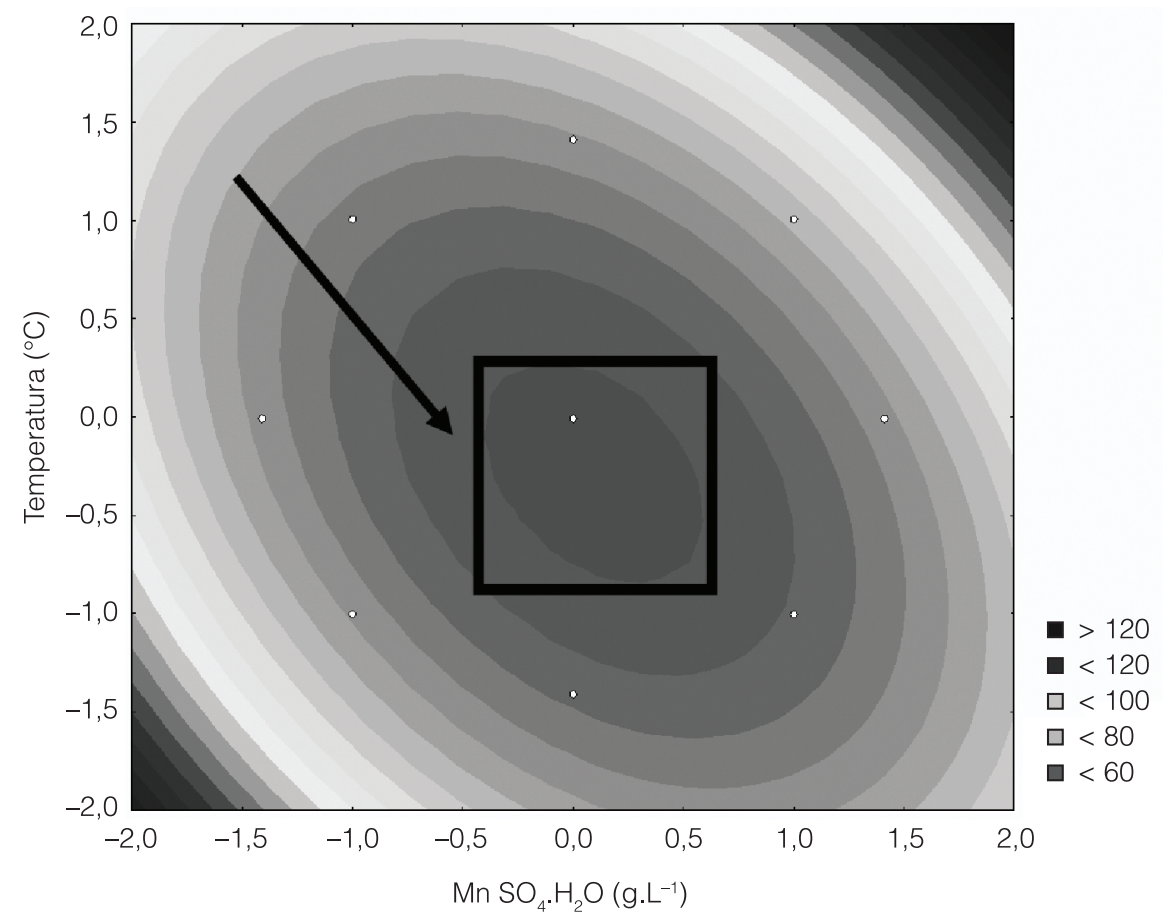

Figura 4. Variação do teor alcóolico em relação aos fatores temperatura e concentração de $\mathrm{MnSO}_{4} \cdot \mathrm{H}_{2} \mathrm{O}$ para a fração "coração".

teor alcoólico (inferior a $60^{\circ} \mathrm{GL}$ ), destacada na Figura 4 , encontra-se delimitada entre os valores do nível de sal de manganês de 0,054 a $0,1 \mathrm{~g} \cdot \mathrm{L}^{-1}$ (níveis $-0,5$ a 0,5), e, para temperatura, entre os valores de 30 e $34{ }^{\circ} \mathrm{C}$ (níveis $-0,5$ a $0,5)$. A faixa descrita compreende a graduação alcoólica do produto destilado que pode ser comercializado como licor de fruta com teor abaixo de $54 \%$ em volume (BRASIL, 2008).

Segundo Aquarone et al. (2001), a adição de sais de magnésio e quantidades de manganês demonstra estimular a ação fermentativa das leveduras. Geralmente, podem ser adicionados na forma de sulfatos, na proporção de 0,2 $\mathrm{g}$ do sal de magnésio e $0,01 \mathrm{~g}$ dos sais de cobalto e manganês.

\section{Conclusões}

Dentre os parâmetros estudados na produção da bebida fermento-destilada a partir do pseudofruto da uva-japão, obtiveram-se os maiores teores de ésteres para a fração "coração". A produção de ésteres foi favorecida pela redução da temperatura e pelo aumento da concentração de $\mathrm{MnSO}_{4} \cdot \mathrm{H}_{2} \mathrm{O}$, sendo maximizada na região compreendida na temperatura de 30 a $24^{\circ} \mathrm{C}$ (níveis $-0,5$ a $-2,0)$ e concentração de $\mathrm{MnSO}_{4} \cdot \mathrm{H}_{2} \mathrm{O}$ de 0,114 a 0,169 g. .-1 (níveis 0,8 a 2,0).

Quanto ao teor de ésteres, pode-se dizer que os valores apresentaram-se muito abaixo dos encontrados na literatura para bebidas fermento-destiladas. $\mathrm{Na}$ etapa de triagem, a quantidade média encontrada foi de 4,00 $\pm 3,25 \mathrm{mg}$ de acetato de etila. $100 \mathrm{~mL}^{-1}$ de álcool anidro, enquanto que, na etapa de otimização, a quantidade média encontrada foi de 2,81 $\pm 3,13 \mathrm{mg}$ acetato de etila. $100 \mathrm{~mL}^{-1}$ de álcool anidro, ambos para a fração "coração".

$\mathrm{O} \mathrm{pH}$ da bebida fermento-destilada a partir da uva-japão não é afetado por variações na temperatura, pelo tempo de fermentação e pelas concentrações de $\mathrm{Mn}^{+2}$ e K+ presentes no meio fermentativo. Para a fração "coração", o pH médio foi de 5,32 e, para a fração "cauda", foi de 3,51.

Para o teor alcoólico presente nas frações "coração" e "cauda", foram observados como fatores mais significativos a temperatura e a concentração de $\mathrm{Mn}^{+2}$. Para a fração "coração", obtiveram-se os menores teores alcoólicos para os níveis intermediários de temperatura e concentração de $\mathrm{Mn}^{+2}$.

\section{Referências}

ALCARDE, R. A.; SOUZA, P. A.; BOSQUEIRO, A. C.; BELLUCO, A. E. S. Cinética de volatilização de componentes secundários da aguardente de cana-de-açúcar durante dupla destilação em alambique simples. Brazilian Journal of Food Technology, Campinas, v. 13, n. 4, p. 271-278, 2010. http://dx.doi. org/10.4260/BJFT2010130400036

AQUARONE, E.; BORZANI, W.; SCHMIDELL, W.; LIMA, U. A. Biotecnologia Industrial: Biotecnologia na Produção de Alimentos. São Paulo: Edgard Blücher, 2001. v. 3, p. 16-17.

ARESTA, M.; BOSCOLO, M.; FRANCO, D. W. Copper (II) Catalysis in Cyanide Conversion into Ethyl Carbamate inspirits 
Influência de parâmetros de processo na obtenção de bebida fermento-destilada de uva-japão (Hovenia dulcis Thunberg) CANCELIER, A. et al.

and Relevant Reactions. Journal of Agricultural and Food Chemical, Washington, v. 49, n. 6, p. 2819-2824, 2001. http:// dx.doi.org/10.1021/jf001346w

BOGUSZ, J. S.; KETZER, D. C. M.; GUBERT, R.; ANDRADES, L.; GOBO, A. B. Composição química da cachaça do Noroeste do Rio Grande do Sul. Ciência e Tecnologia de Alimentos, Campinas, v. 26, n. 4, p. 793-798, 2006. http://dx.doi. org/10.1590/S0101-20612006000400013

BRASIL. Ministério da Agricultura Pecuária e Abastecimento. Decreto $n^{\circ} 2.314$, de 4 de setembro de 1997. Dispõe sobre a padronização, a classificação, o registro, a inspeção, a produção e a fiscalização de bebidas. Diário Oficial da República Federativa do Brasil, Brasília, DF, 1997. Seção 4, cap. 4, art. 91b.

BRASIL. Ministério da Agricultura Pecuária e Abastecimento. Portaria $n^{\circ}$ 62, de 23 de abril de 2008. Regulamentos técnicos para a fixação dos padrões de identidade e qualidade para licor. Diário Oficial da República Federativa do Brasil, Brasília, DF, 25 abr. 2008.

CARDEAL, Z. L.; SOUZA, P. P.; SILVA, M. D. R. G.; MARRIOTT, P. J. Comprehensive two-dimensional gas chromatography for fingerprint pattern recognition in cachaça production. Talanta, Washington, v. 74, n. 4, p. 793-799, 2008. PMid:18371711. http:// dx.doi.org/10.1016/j.talanta.2007.07.021

CARVALHO, P. E. R. Ecologia, silvicultura e usos da uva-do-japão (Hovenia dulcis Thunberg). Colombo: EMBRAPACNPFlorestas, 1994. 24 p. (Circular Técnica, n. 23).

DANTAS, H. J.; VILAR, F. A.; SILVA, F. L. H.; SILVA, A. S. Avaliação da influência da velocidade de destilação na análise físicoquímica de aguardente de cana-de-açúcar. Revista Brasileira de Produtos Agroindustriais, Campina Grande, v. 9, n. 2, p. 101-109, 2007

GARCIA-LLOBODANIN, L.; ACHAERANDIO, I.; FERRANDO, M.; GUELL, C.; LOPEZ, F. Pear distillates from pear juice concentrate: Effect of lees in the aromatic composition. Journal of Agricultural and Food Chemistry, Washington, v. 55, n. 9, p. 3462-3468, 2007. PMid:17394335. http://dx.doi.org/10.1021/ jf0633589

INSTITUTO ADOLFO LUTZ - IAL. Normas Analíticas do Instituto Adolfo Lutz. 3. ed. São Paulo: IMESP, 2005. v. 1: Métodos físicos e químicos para análise de alimentos. 1020 p.
LIMA, A. J. B.; GUIMARÃES, L. G. L.; LIMA, J. M. S.; NELSON, D. L. Efeito de substâncias empregadas para remoção de cobre sobre o teor de compostos secundários da cachaça. Química Nova, São Paulo, v. 32, n. 4, p. 845-848, 2009. http://dx.doi. org/10.1590/S0100-40422009000400004

MARRA, B. M. Avaliação de Parâmetros Operacionais e Remoção de Congêneres Secundários Tóxicos para a Melhoria da Qualidade da Cachaça de Alambique. 2008. 88 f. Tese (Doutorado em Biologia Celular)-Instituto de Ciências Biológicas, Universidade de Brasília, Brasília, 2008.

MIRANDA, M. B.; MARTINS, N. G. S.; BELLUCO, A. E. S.; HORII, J.; ALCARDE, A. R. Perfil físico-químico de aguardente durante envelhecimento em tonéis de carvalho. Ciência e Tecnologia Alimentos, Campinas, v. 28, p. 84-89, 2008. http://dx.doi. org/10.1590/S0101-20612008000500014

RECHE, R. V.; LEITE, A. F.; SILVA, A. A.; GALINARO, C. A.; OSTI, R. Z.; FRANCO, D. W. Influence of type of distillation apparatus on chemical profiles of Brazilian cachaças. Journal of Agricultural and Food Chemistry, Washington, v. 55, n. 16, p. 6603-6608, 2007. PMid:17629298. http://dx.doi.org/10.1021/ jf0704110

ROSA, C. A.; SOARES, A. M.; FARIA, J. B. Cachaça production. In: INGLEDEW, W. M. (Ed.). The Alcohol Textbook. 5th ed. Nottingham: Nottingham University Press, 2009. p. 484-497.

SAERENS, S. M. G.; DELVAUX, F.; VERSTREPEN, K. J.; VAN DIJCK, P.; THEVELEIN, J. M.; DELVAUX, F. R. Parameters Affecting Ethyl Ester Production by Saccharomyces cerevisiae during Fermentation. Applied and Environmental Microbiology, Washington, v. 74, n. 2, p. 454-461, 2008. PMid:17993562 PMCid:2223249. http://dx.doi.org/10.1128/AEM.01616-07

SOUZA, M. D. C. A.; DEL MASTRO, N. L. Efeito da radiação gama na aguardente de cana-de-açúcar. Brazilian Journal of Food Technology, Campinas, v. 7, n. 1, p. 9-15, 2004.

SUTTISRI, R.; LEE, I. S.; KINGHORN, A. D. Plant-derived triterpenoid sweetness inhibitors. Journal Ethnopharmacology, Leiden, v. 47, p. 9-26, 1995. http://dx.doi.org/10.1016/03788741(95)01248-C

TORIJA, M. J.; ROZEZ, N.; POBLET, M.; GUILLAMON, J. M.; MAS, A. Effects of fermentation temperature on de strain population of Saccharomyces cerevisiae. International Journal of Food Microbiology, Torino, v. 80, p. 47-53, 2003. http://dx.doi. org/10.1016/S0168-1605(02)00144-7 